\title{
Nondestructive Examination Technologies for Inspection of Radioactive Waste Storage Tanks
}

M. T. Anderson

D. C. Kunerth

J. R. Davidson

Published August 1995

\author{
Idaho National Engineering Laboratory \\ Materials Physics Department \\ Lockheed Idaho Technologies Company \\ Idaho Falls, Idaho 83415
}

Prepared for the

U.S. Department of Energy

Assistant Secretary for Environmental Management

Under DOE Idaho Operations Office

Contract DE-AC07-94ID13223

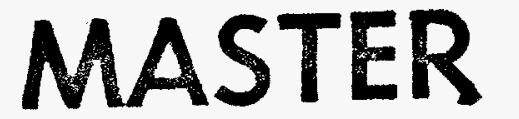





\section{DISCLAIMER}

Portions of this document may be illegible in electronic image products. Images are produced from the best available original document. 


\begin{abstract}
The evaluation of underground radioactive waste storage tank structural integrity poses a unique set of challenges. Radiation fields, limited access, personnel safety, and internal structures are an end effector (EE) on a robotic arm. The purpose internal surfaces, a sensor suite must be deployed as modes of the tanks, rank the viabilitilure select a technology for initial EE implementation implementation, and project future needs for NDE EE sensor suites.
\end{abstract}

\title{
DISCLAIMER
}

This report was prepared as an account of work sponsored by an agency of the United States Government. Neither the United States Government nor any agency thereof, nor any of their employees, makes any warranty, express or implied, or assumes any legal liability or responsibility for the accuracy, completeness, or usefulness of any information, apparatus, product, or process disclosed, or represents that its use would not infringe privately owned rights. Reference herein to any specific commercial product, process, or service by trade name, trademark, manufacturer, or otherwise does not necessarily constitute or imply its endorsement, recommendation, or favoring by the United States Government or any agency thereof. The views and opinions of authors expressed herein do not necessarily state or reflect those of the United States Government or any agency thereof. 


\section{CONTENTS}

ABSTRACT $\ldots \ldots \ldots \ldots \ldots \ldots \ldots \ldots \ldots \ldots \ldots \ldots \ldots \ldots \ldots \ldots \ldots \ldots \ldots \ldots$

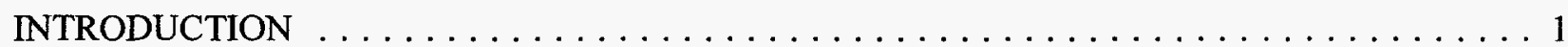

MATERIALS DEGRADATION CONCERNS $\ldots \ldots \ldots \ldots \ldots \ldots \ldots \ldots \ldots \ldots \ldots \ldots$

POTENTIAL NONDESTRUCTIVE EXAMINATION TECHNOLOGIES $\ldots \ldots \ldots \ldots \ldots$

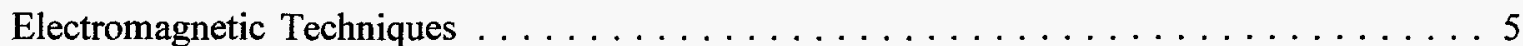

Eddy Current Techniques $\ldots \ldots \ldots \ldots \ldots \ldots \ldots \ldots \ldots \ldots \ldots \ldots \ldots \ldots \ldots \ldots \ldots$

Magnetic Flux Measurements $\ldots \ldots \ldots \ldots \ldots \ldots \ldots \ldots \ldots$

Alternating Current Field Measurement (ACFM) $\ldots \ldots \ldots \ldots \ldots \ldots$

Radiographic Techniques $\ldots \ldots \ldots \ldots \ldots \ldots \ldots \ldots \ldots \ldots \ldots \ldots \ldots \ldots$

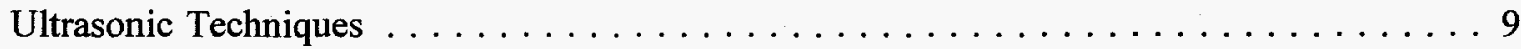

CURRENT ACTIVITIES $\ldots \ldots \ldots \ldots \ldots \ldots \ldots \ldots \ldots \ldots \ldots \ldots \ldots \ldots$

FUTURE NDE ENHANCEMENTS $\ldots \ldots \ldots \ldots \ldots \ldots \ldots \ldots \ldots \ldots \ldots \ldots \ldots$

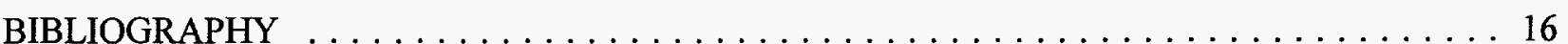

Attachment A - VENDOR FEASIBILITY STUDY $\ldots \ldots \ldots \ldots \ldots \ldots \ldots \ldots \ldots \ldots$ 



\section{Nondestructive Examination Technologies for Inspection of Radioactive Waste Storage Tanks}

\section{INTRODUCTION}

By-products of nuclear fuel reprocessing and other special nuclear operations are stored in large underground tanks at various DOE sites. Most of these tanks were fabricated and placed in service many years ago to support cold war defense activities. The principal concern with these storage tanks is their ability to perform as designed - to contain the hazardous materials. This required the tanks to remain leak-tight and structurally intact well beyond their intended service lifetimes. Most of the DOE tanks were not equipped with quantitative leakage measurement systems. The large volumes of stored waste involved and regulatory requirements to monitor minute levels of leakage make it increasingly important to assess tank structural integrity with regard to mitigating potential leakages. Several leakage events have occurred, prompting DOE to begin remedial actions to ensure that tank integrity is maintained and the surrounding environment is protected. The materials stored in these tanks contain dangerous chemicals and/or radioactive fission products, further complicating the maintenance and eventual decommissioning of the tanks.

Practically all of the remediation work will entail the use of remote procedures due to the hazardous nature of the stored materials. A robotic, light duty utility arm (LDUA) is being developed to support inspection of the tanks during the remediation process. The LDUA will be used in conjunction with several "end effectors" to assess the waste and the structural integrity of the tanks. One of these end effectors will use nondestructive examination (NDE) methods to evaluate the tank interior for evidence of service-induced degradation that might impact the tank's design function or planned remediation activities. This report is intended to provide a basis for developing an NDE technology for use with the LDUA. Materials degradation issues related to DOE underground storage tanks (USTs), the near-term use of proven NDE technologies to detect degradation, and development of enhanced methods of acquiring large area volumetric information to support future tank integrity assessments are discussed. 


\section{MATERIALS DEGRADATION CONCERNS}

The tanks are of welded construction, fabricated from either carbon or stainless steel plate with nominal thicknesses ranging from $1 / 4$ to $3 / 4$ inches. They typically are short, right vertical cylinders with a flat bottom and a domed top. Access for inspections is limited to "risers," essentially pipes, of various diameter that extend from the dome of the tank up to grade level. Depending on the specific tank design and function, internal structures such as cooling coils and support columns must be considered when examining interior surfaces.

Structural integrity is influenced by both operating stress levels and materials degradation. These phenomena are interdependent: for a given material thickness, a decrease in "strength" due to degradation will cause an increase in hydraulic-induced and dead load local stresses. Degradation can be broadly defined to include any process that produces one or more of the following conditions: 1) a decrease in material thicknesses beyond nominal design values, 2) initiation and growth of flaws, 3) a detrimental change in metallurgical and/or mechanical properties. Metallurgical and mechanical properties are not likely to be affected by the moderate ambient temperatures, type(s) of radiation present, or relatively low operating stress levels of the tanks. Therefore, material thickness changes and flaw initiation/growth are the primary concerns relative to potential materials degradation in DOE underground storage tanks.

For the environmental conditions found in these tanks, corrosion is the dominant mechanism of degradation. Chemical stratification, elevated local temperatures, and residual stresses near the welds are some of the variables that can influence corrosion rates. The actual amounts of corrosion degradation are unknown because most of the tanks have not been adequately examined during their service lifetimes. For a limited number of tanks, corrosion testing via coupons placed in the actual waste has been performed; however, unknown conditions, e.g. residual welding stresses or localized chemistry effects, may promote degradation not predicted by the in-tank tests.

Several specific corrosion mechanisms have been identified that may impact tank structural integrity. These are:

- Uniform, or general, corrosion - This is the most common form of corrosion and is caused by chemical and electrochemical reactions, or mechanical actions in the case of erosion, that occur 
over a large exposed surface area and uniformly reduce wall thickness. Typically, uniform corrosion has a high rate until a passivated material layer is formed, after which the rate progressively decreases to some minimum value that continues over the life of the component. However, significant fluctuations in environmental conditions may remove portions of the passivated material, exposing surfaces that are again subject to rapid corrosion rates. Uniform corrosion can result in an overall thinning of the material until ductile failure eventually occurs due to design stresses being exceeded.

- Pitting - This form of corrosion results from an extremely localized attack that initially causes small, discrete pits, or holes, in the material. If allowed to continue, pitting may eventually penetrate the wall, resulting in leakage. In addition, small pits, while not compromising the overall load-bearing function of the tanks, may provide initiation sites for other corrosion/cracking mechanisms.

Crevice corrosion - This is an aggressive process that occurs within crevices, or on surface areas that are separated from the main body of the corrosive medium. The separation can be due to the geometry of the component, e.g., holes, gasket surfaces, bolt or rivet heads, lap joints, or to welding anomalies such as incomplete fusion, quench-cracks, and undercut. In the case of the underground storage tanks, a more likely cause for crevice corrosion would be areas where non-metallic surface deposits harden to form a porous shield, and create a localized, chemically stagnant environment. Areas with high potential for this type of corrosion are at or near the bottom of tanks where a sludge may be present. Typical crevice corrosion rates are orders of magnitude higher than those of uniform corrosion.

Stress-corrosion cracking - This form of degradation is caused by the interaction of tensile stress with a corrosive environment. Stress-corrosion cracking (SCC) usually occurs where there are highly localized stress phenomena such as residual stresses in the heat-affected zones (HAZ) of welds or stress risers caused by metallurgical anomalies and fabrication techniques/conditions. SCC has been observed even when the local stress phenomena are somewhat low relative to the overall operating stress levels of the component. In addition, SCC can occur when the medium is relatively benign; it has been documented in commercial nuclear systems exposed to deionized water with moderate levels of dissolved oxygen and $\mathrm{pH}$ values ranging from 5 to 7 . SCC paths may either be intergranular or transgranular, depending on the specific material and environmental conditions. Generally, SCC is characterized by extremely fine, branched cracking 
in localized areas while the remainder of the material is virtually unaffected. SCC propagates in a direction nearly perpendicular to the maximum principal tensile stress. SCC is the most intense form of localized corrosion and generally the most difficult degradation mechanism to detect. It can have serious consequences because it may occur within the design operating stress range.

In summary, conditions within DOE underground storage tanks may support any, or all, of the corrosion mechanisms previously described. Knowledge of the characteristics and geometric orientation of these forms of degradation is paramount in choosing and optimizing nondestructive examination methods used for determining the structural integrity of the tanks. 


\section{POTENTIAL NONDESTRUCTIVE EXAMINATION TECHNOLOGIES}

Numerous NDE methods have potential for use in characterizing the general condition of the USTs. However, due to budget and schedule limitations, it was decided to investigate those technologies that are currently used in standard industrial applications and are expected to meet the minimum requirements for this task. This avoids basic developmental efforts.

\section{Electromagnetic Techniques}

Electromagnetic test methods have been used for a number of years to perform various tasks, including quantitative material property measurements (e.g. conductivity, permeability), dimensional measurements (e.g. coating thickness, wall thickness), and detection and characterization of discontinuities (e.g. cracks, pits, porosity, corrosion). The electromagnetic properties that are key to these inspection technologies, because they directly correspond to physical, structural, and mechanical characteristics, are electrical conductivity, electrical permittivity, and magnetic permeability. For example, conductivity/permeability is a function of metal alloy constituents and so can be used for alloy sorting. The conductivity/permeability/permittivity of a coated substrate can be compared to those of test standards to determine coating thickness. Local bulk conductivity/permeability is disrupted by discontinuities and thus can be used for crack detection.

The primary electromagnetic techniques use eddy currents, magnetic particles, magnetic flux measurements, microwave measurements, and potential drop measurements (including Alternating Current Field Measurements, ACFM). Of these, eddy current, magnetic flux, and ACFM measurements are deemed applicable to storage tank wall inspection. They are suitable for detecting and characterizing cracks, pits, and wall thinning due to corrosion in both stainless steel and carbon steel.

\section{Eddy Current Techniques}

Eddy current testing is based on the generation of eddy currents through inductive coupling of a test coil and an electrically conductive sample. These currents can be sensed via modulation of the primary magnetic fields by the secondary eddy current fields or independently with external sensing coils. The primary electromagnetic properties sensed by eddy current techniques are electrical 
conductivity and magnetic permeability. Any material or physical condition (e.g. cracks or pits) that locally affects one or both of these properties can be sensed and characterized. Sample geometry, such as edges and thickness, affects inductive coupling and is the means through which wall thinning can be detected.

Three different types of eddy current testing may be useful in tank wall inspection:

1) Continuous Wave ( $C W$ ) Techniques - these techniques use one or a limited number of continuous sine waves to drive the test coil. CW eddy current testing is a mature, well understood technology that is widely used by industry and well supported by various commercial vendors. However, the information available is limited by the number of frequencies used. Assuming small signals with linear approximation, the number of separate parameters that can be detected is twice the number of test frequencies employed (i.e., amplitude and phase for each frequency). The depth of penetration for $\mathrm{CW}$ techniques is limited by test frequency, test material conductivity and permeability, and drive signal amplitude. In highly permeable materials, such as iron, penetration is significantly limited. In general, $\mathrm{CW}$ eddy current technologies are able to characterize cracks and pits and to measure thickness in stainless steel (low conductivity and permeability) but are only useful for near-surface cracks and pits in carbon steel (high permeability).

2) Pulsed Eddy Current (PE) Techniques - these techniques use pulsed drive signals instead of continuous sine waves. The pulses are broad band in nature, thus providing multifrequency information, and greater penetration is achieved because the lower duty cycle of a pulse permits greater loading of the drive coil. Defect location can be determined from time-of-flight information, and signal processing can be directly built into the drive signal via pulse shaping. However, signal interpretation and modeling are more complex and instrumentation is complicated in design. The attributes that may be of value for tank inspection are increased penetration, which allows measuring the thickness of heavy section stainless steel walls; time-offlight measurement for location of defects (i.e. differentiation of near side/far side defects based on time); and real-time signal processing via pulse shaping to minimize the effects of conflicting test variables. Although $\mathrm{PE}$ has better penetration capabilities than $\mathrm{CW}$, they are not sufficient for carbon steel. 
3) Remote Field Eddy Current (RFEC) Techniques - in essence, remote field eddy current testing is a low frequency $\mathrm{CW}$ technique that uses a unique arrangement of driver/sensor coils to detect remote $^{a}$ flux lines that have traversed the thickness of the test piece twice, through and back. RFEC can inspect ferrous or nonferrous materials with equal sensitivity to near side and far side defects and is quite sensitive to wall thickness, demonstrating a linear relationship between wall thickness and phase lag. As originally developed and implemented, RFEC is limited to pipe geometries. However, this limitation may be overcome by current work addressing planar and near-planar geometries. RFEC's greatest asset for tank wall inspection is the ability to fully penetrate highly permeable material such as carbon steel to provide information on both sides of the test region. RFEC is a mature technology that is supported by various commercial vendors.

\section{Magnetic Flux Measurements}

Magnetic flux measurements are based on the interaction of highly permeable test materials (e.g., carbon steel, not stainless steel) with lines of magnetic flux. Analogous to eddy current testing, material electromagnetic properties and physical condition affect both the quantity and path of the flux lines. For example, the most commonly applied flux measurement technique, magnetic particle testing, is based on the alignment of iron filings with flux diversions at a crack or other discontinuity of magnetic permeability. Of the magnetic flux inspection techniques available, only magnetic reluctance measurements and magnetic flux leakage are deemed to have potential for tank wall inspection.

1) Magnetic Reluctance Measurements - these measurements are performed by monitoring the flux density of a magnetic circuit that includes part or all of the test sample. Variations in sample thickness or cracks result in variations of the flux density. Unfortunately, variations in density, microstructure, or other material conditions that disrupt permeability, e.g. welds, also affect the flux density. In general, this approach has similar capabilities to basic $\mathrm{CW}$ eddy current techniques but is limited to near-surface carbon steel defects. Magnetic reluctance techniques are not commonly applied and limited commercial support is available.

a. In pipe geometries, the remote field zone starts approximately 2 inside pipe diameters away from drive coil. 
2) Magnetic Flux Leakage (MFL) Measurements - as in other magnetic methods, cracks, inclusions, welds, wall thinning, etc. disrupt the permeability of the test piece and alter the path and amplitude of the magnetic flux introduced into the sample. For MFL, the flux that "leaks" from the surface is measured. In general, flux leakage measurements could be considered as quantifying magnetic particle measurements of discontinuities by replacing iron filings with magnetic field detectors such as coils, hall probes, and magnetodiodes. This technology has been used for the inspection of above ground storage tanks and may be adaptable for remote inspection of below ground tanks; power requirements, drive coil size, and magnetic forces may be limiting factors. Magnetic flux leakage is widely used by industry and supported by a variety of commercial vendors.

\section{Alternating Current Field Measurement (ACFM)}

ACFM is an electromagnetic technique that is used to detect and characterize surface and nearsurface defects in both ferrous and nonferrous materials. A uniform alternating current is generated in the surface of the test piece and the localized surface potential drop or localized external magnetic field variations are measured via magnetic field sensors. The induced current is confined to the surface by the skin effect, which is governed by test frequency and sample conductivity and permeability. Both contacting and noncontacting versions of this technique are used. Because the induced current follows the sample's surface, careful implementation of this technique allows geometry effects, such as from weld beads, to be minimized. Defects such as cracks and pits can be analytically characterized based on sample characteristics and an assumed crack/pit geometry. As currently practiced, this technology will detect cracks and pits but is not well suited to thickness measurements in carbon or stainless steel. ACFM is used and commercially supported in Europe for the inspection of oil platforms but is not widely implemented in the U.S.

\section{Radiographic Techniques}

Radiography, one of the earliest NDE methods, has been used extensively to examine components for fabrication flaws and, to a lesser degree, to detect service-related defects such as cracks or pitting. In radiography, one side of a material is generally exposed to either $\mathrm{x}$ - or gamma radiation that is detected on the other side. Standard industrial radiographic testing (RT) methods use radiographic film, similar to photographic film, to record the image produced as the $\mathrm{x}$ - or gamma 
energy penetrates the specimen. Areas in the material that absorb less penetrating radiation, i.e., internal voids due to cracks and other fabrication flaws, or surface-breaking anomalies such as pits and erosion/corrosion, are darker on the film than the surrounding material. After developing the film, one has a "shadow picture" of the part being examined. Depending on the intensity of radiation, and the orientation of the flaw, RT can be used to detect very fine flaws. Detectors other than radiographic film are also useful in specific applications. The limiting condition of RT is the need to access both sides of the material to be examined; synchronized robotic fixtures on the inner and outer surfaces of the USTs would be necessary for standard radiographic implementation.

\section{Ultrasonic Techniques}

One of the primary industrial inspection techniques is ultrasonic testing (UT). This volumetric examination method directs high frequency sound waves into a material at the proper orientations for anomalies (cracks, pitting, corrosive wall-thinning) to reflect the sound energy, indicating their locations and relative size. A UT pulse is normally generated using a piezoelectric crystal that is caused to expand and contract at a central frequency (normally in the megahertz regime) by application of electrical impulses. By placing this ultrasonic transducer in contact with the material to be examined, acoustic vibrational energy is transferred. The transfer of sound energy is facilitated by a coupling medium (generally liquid) between the transducer and the material to be examined. The coupling medium excludes the surrounding air, which would attenuate, or dampen out, the ultrasonic sound energy.

Sound energy propagates through the material by causing the individual atoms in the lattice structure to vibrate; this vibration is translated to the following layer of atoms, and so on until the sound reaches a reflecting medium such as an anomaly, or the back surface of the material. As the sound travels through the material, it decreases in energy due to absorption and scattering, which are functions of the mode of propagation and wavelength and amplitude of the initial pulse. Knowing these variables, as well as the relative sound velocity in a particular material, one may predict and measure the time of flight necessary for the acoustic wave to traverse the part. This enables the examiner to calibrate an instrument so that it is capable of measuring the location of reflectors within the material and to observe the amplitude and echo dynamics of the reflected sound energy. 
UT is an accepted technique for detection of cracking and pitting and for measuring wall thinning due to corrosion. However, several variables and/or limitations must be considered prior to deploying this method for examining the USTs; these are:

1) The need to physically couple a transducer to the tank wall. A coupling liquid would be required to be continuously applied to the tank wall. Dry coupling is feasible (see discussion under Current Activities Section in this report), but has not yet been attempted in such an application where multiple forms of degradation may occur.

2) Multiple angles of sound within the material are typically required to characterize cracking and pitting. This is achieved via multiple or articulated transducers, which complicates the design of potential scanning assemblies.

3) The detection/characterization limits for discrete flaws originating on the inner surface of the tanks are unclear. This is especially true in the area of "as-welded" joints where weld crowns may interfere with transducer scanning motions on the surface of the tank wall. 


\section{CURRENT ACTIVITIES}

For FY-95, the NDE end effector development effort is aimed at providing a tool for future verification of the structural integrity of several high level waste storage tanks located at the Chemical Processing Plant (CPP) on the Idaho National Engineering Laboratory (INEL). The CPP tanks are constructed of welded stainless steel plates, with nominal wall thicknesses in the 3/16- to 5/16-inch range. Based on the exceptional operating history, including a marked absence of detected leakage and an existing corrosion testing program, it is believed that the CPP tanks are in relatively good condition with respect to the degradation mechanisms previously described.

Three NDE technologies were considered for the initial demonstration end effector, ACFM, dry-coupled UT, and continuous wave ET. The attractive features of these technologies are that 1) nothing (e.g., water, couplant, etc.) would have to be added to the tanks; 2) their essential variables are well understood, so developmental efforts would be minimal; and 3) they can be remotely deployed.

The ACFM method exhibited an excellent potential for detecting inner surface-breaking flaws, while being relatively insensitive to distortions caused by weld geometries. However, this technology was not considered immediately viable due to several factors. The first of these is that ACFM cannot measure wall thicknesses in the range needed for the CPP tanks. Due to the nonferromagnetic nature of 304 stainless steel, and to other fixed system parameters, current field density decreases as a function of penetration into the material. This results in a field penetration less than the nominal tank wall thickness of 1/4-inch. Another problem for ACFM is the lack of acceptance by U.S. regulatory authorities and standards institutes. This method is virtually unknown in U.S. industry, and an extensive and prolonged qualification effort could be required to gain an appropriate level of recognition prior to implementation in a nuclear application. Finally, given FY-95 budget constraints, the cost of radiation-hardening various electronics in the detector array assembly precludes using this technology in the demonstration end effector.

The dry-coupled UT method uses a special, integral wheel-mounted probe arrangement with a pliant membrane, such as natural rubber, to provide a sound coupling path for the acoustic wave energy. UT technology is highly accurate in measuring wall thickness, and is the recognized industry standard for crack detection and sizing for a wide range of component inspections. However, it is unclear whether the dry-coupled UT method would be sufficiently sensitive for flaw detection/sizing 
when applied from the surface on which the flaw is located. Flaw detection and sizing using wheeltype probes necessitates a pitch-catch, forward tip diffraction technique that requires a dual set of sensors. The overall size of this tandem wheel arrangement would limit sound path accessibility to the weld HAZ due to weld crown geometry and would obscure the visual image of the scanning area. Until such time as these issues can be resolved, remote dry-coupled UT is not feasible for inspecting the welded regions of the tanks.

The third technology investigated this year was continuous wave ET. This technique, more commonly called eddy current testing, is used for detecting service-related degradation in relatively thin-wall, nonferromagnetic materials. Typical applications include heat exchanger tubing and various aircraft structural and engine components. At the request of the INEL, a commercial ET vendor performed a feasibility study to determine the technology's ability to detect cracks located in the HAZ of welds, to detect pitting both on the weld and base material, and to make wall thickness measurements in areas of base material. The study, detailed in Appendix A, was performed using a welded 304 stainless steel plate fabricated to simulate the CPP tank walls.

The feasibility study indicated that crack and pit detection in the INEL sample plate was relatively straightforward using available off-the-shelf components. When specific coil configurations are optimized, and an appropriate set of calibration standards and reference curves applied, sizing of these flaws is limited only by the minimum overall size of the ET coils. In addition, wall thinning due to corrosion/erosion appeared to be measurable. A simplified test was performed by nulling a probe on nominal material, then placing the sensor on each of successively thinned steps to observe changes in the balanced operating point. This test showed discrete changes ranging from 0.5 to 3.0 screen divisions for respective thinned regions. A continuous scan over the set of thinned steps was then performed, and also resulted in significantly different signal responses from the nominal to thinnest wall regions. Some development is necessary to optimize the technique and determine measurement accuracy; however, this preliminary information indicates that ET is a viable methodology for deployment in the CPP tanks.

The FY-95 strategy is to procure an ET data acquisition/analysis system capable of running on a UNIX work platform, with integrated scanner control software and hardware that includes a scanner with on-demand $\mathrm{X}$-axis or $\mathrm{Y}$-axis scanning and a modular suite of surface-compliant ET sensors. It is expected that these items, with the exception of the scanner assembly and sensor module, exist as standard off-the-shelf components so minimum development will be required. The procured system 
will be mounted into an INEL-developed housing that will accommodate end effector misalignment up to 15 degrees during wall contact, and will control scanner offset to a flat plate within 0.060 inches across the entire scanner front surface. The housing will also include a video camera/lens system and provide other mechanical interfaces and status indicators to enable optimum positioning of the end effector on the tank wall.

A system capabilities plan will be developed to provide a basis for comparison of the ET system and future NDE technologies. Initially, the thresholds and limits of flaw detection/sizing will be defined via data acquisition and analysis of a series of flaws in sample plates designed to simulate actual tank walls at CPP. This information will be documented and used for benchmarking, as well as to provide insights for future enhancements that may be needed to facilitate deployment or optimize the overall inspection. 


\section{FUTURE NDE ENHANCEMENTS}

The NDE end effector developed during this fiscal year will meet the functional requirements set forth for use in the CPP tanks. However, improvements to the system are necessary for deployment at other DOE facilities and to allow more efficient use in the future. The first of these is to establish capabilities for carbon steel, or other ferromagnetic materials. The CPP tanks are 300 series stainless steel, therefore, electromagnetic permeability changes are minimized. The continuous wave eddy current technique relies on constant permeability for induced field stability. However, new techniques such as pulsed and remote field eddy current show promise for use on ferromagnetic materials, e.g. the carbon steel tanks located at Hanford. In FY-96, the capabilities of these new techniques will be investigated, and potential design modifications to the existing end effector determined, for application on ferromagnetic materials.

Another item to be addressed in FY-96 is the planned deployment methodology, specifically the in-tank inspection sequence. The current NDE end effector is designed to land on the tank wall such that a qualified operator can scan an area that has been selected based on remote visual inspection. The main premise of this methodology is that degradation, if present, will be visually detectable. While this approach may be adequate for areas of excessive corrosion and pitting, it will not detect more insidious forms of inservice degradation, i.e., stress-corrosion or fatigue cracking. Furthermore, requiring an operator to make numerous detailed scans of small (4 in. $\mathrm{x} 4 \mathrm{in}$.) areas to assess the overall integrity of the tank is inefficient, expensive, and probably inconclusive. A large area scan, or "fly-by" technique, is necessary for the periodic examination of these tanks. The INEL will investigate potential technologies that would allow significant sections of the tank wall to be inspected relatively quickly, and areas of potential degradation noted for subsequent characterization by the current end effector.

Finally, while it is believed that the new NDE system will provide a acceptable characterization of inservice degradation at CPP, unknown variables may limit the capabilities of continuous wave ET for certain "as-found" conditions. For this reason, we propose to reexamine the limitations noted in our preliminary investigation of UT. Ultrasonics is a valuable method for crack detection and sizing, and would provide a means for corroborating indications found with the eddy current technique. Additionally, UT is the industry standard for determining remaining wall thickness of components. We plan to determine the feasibility of deploying UT alongside the eddy current 
sensors in the new end effector so that multiple capabilities may be employed to minimize the effect of unknown material conditions. 


\section{BIBLIOGRAPHY}

Amos, D. M., “The Truth about Magnetic Flux Leakage as Applied to Tank Floor Inspection," Proc. of ASNT's International Chemical and Petroleum Industry Inspection Technology (ICPIIT) IV Topical Conference, Houston, TX, June 19-22, 1995, pp. 232-236.

Blitz, J., Electrical and Magnetic Methods of Nondestructive Testing, Adam Hilger, Philadelphia, 1991.

Collins, R., W. D. Dover, and D. H. Michael, Research Techniques in Nondestructive Testing, "The Use of a. c. Field Measurements For Non-Destructive Testing," ed. R. S. Sharpe, Vol. 8, Academic Press, New York, 1985, pp. 211-267.

Crostack, H. A., W. Oppermann, and J. Nehring, "Application of Correlated Pulses (CS-Technique) to Eddy Current Testing," MaterialPrufung, Vol. 26, No. 1-2, January-February 1984, pp. 16-20.

Functional Specifications, Eddy Current Scanner System for the Nondestructive Examination End Effector, Idaho National Engineering Laboratory, March 1995.

Harman, D. G., Tank Waste Remediation System - Tank System Integrity Assessments Program Plan, WHC-SD-WM-AP-017, Rev. 1, June 1994.

Libby, H. L., Introduction to Electromagnetic Nondestructive Test Methods, Wiley-Interscience, New York, 1971.

Libby, H. L., "Multiparameter Eddy Current Concepts," in Research Techniques in Nondestructive Testing, Chap. 11, ed. R. S. Sharpe, Vol. 1, Academic Press, New York, 1970, pp. 345-382.

Lugg, M. C., "An Introduction to ACFM," Technical Software Consultants Ltd., Document MCL/0655, October 1992.

Nondestructive Testing Handbook, Vol. 4, "Electromagnetic Testing", 2nd Ed., Ed. R. C. McMaster, P. McIntire, M. L. Mester, American Society of Nondestructive Testing, 1986. 
Palanisamy, R., "Remote-Field Eddy Current Testing: A Review," Review of Progress in Quantitative Nondestructive Evaluation, Vol. 7A, (1988), pp. 157-164.

Sather, A., "Investigation into the Depth of Pulsed Eddy-Current Penetration," Eddy Current Characterization of Materials and Structures, ASTM STP 722, eds. G. Birnbaum and G. Free, American Society for Testing Materials, 1981, pp. 374-386.

Schwenk, E. B., Tank Wall Measurement Technical Study and Specification, WHC-SD-W151-ES-002, Rev. 0, June 1992.

Shurrab, M. S., et al., Parametric Studies to Support Inspection Criteria of the Hanford Site Double-Shell Waste Storage Tanks, WHC-EP-0508, Vol. 1, September 1991.

Sun, Y. S., S. Udpa, W. Lord, and D. Cooley, "A Remote Field Eddy Current, NDT Probe for the Inspection of Metallic Plates," Proc. of ASNT's International Chemical and Petroleum Industry Inspection Technology (ICPIIT) IV Topical Conference, Houston, TX, June 19-22, 1995, pp. 155-158.

Waidelich, D. L., "Pulsed Eddy Currents," in Research Techniques in Nondestructive Testing, Chap. 12, ed. R. S. Sharpe, Vol. 1, Academic Press, New York, 1970, pp. 383-416.

Wailelich, D. L., "Pulsed Eddy Current Testing of Steel Sheets," Eddy Current Characterization of Materials and Structures, ASTM STP 722, eds. G. Birnbaum and G. Free, American Society for Testing Materials, 1981, pp. 367-397.

You, Z., and D. Bauer, "Combining Eddy Current and Magnetic Flux Leakage for Tank Floor Inspection," Materials Evaluation, Vol. 52, No. 7, 1994, pp. 816-818. 
Attachment A

\section{VENDOR FEASIBILITY STUDY}

A-1 
March 15, 1995

Mr. Michael Anderson

Idaho National Engineering Laboratory (INEL)

P.O. Box 1625

Idaho Falls, ID 83415-2209

Re: Feasibility Study for Scanning High Level Radiation Waste Storage Tanks

Dear Mr. Anderson,

Following is brief outline of the work performed to date as a "no cost feasibility study" for INEL.

\section{Introduction:}

INEL contacted Zetec in an attempt to determine the feasibility of testing 300 Series Stainless Steel High Level Rad Waste Storage Tanks utilizing Eddy Current Testing Technology. At no cost to INEL, Zetec performed a feasibility study, developed test parameters and submitted a preliminary report outlining the results. Based on the favorable results of the initial feasibility study, Zetec has made specific recommendations to INEL regarding coil selection, instrumentation and technique.

\section{Data Acquisition Process:}

All data has been collected utilizing Zetec's MIZ-40A Eddy Current Tester. The MIZ-40A is a multi-frequency instrument which allows excitation of a coil at up to 4 frequencies at a time. Test frequencies were chosen to obtain the best results for specific areas of concern. Specific setup information can be noted on the attached graphics. To accommodate all the test requirements set forth by INEL, 3 separate tests were performed. In conjunction to the flaws in the standard provided by INEL, Zetec machined a set of round bottom holes and steps into the sample for further testing. Figure 6 is a drawing of the standard used. 


\section{Weld Toe Region Cracking:}

The initial test was for the detection of cracks and porosity located in the toe of the weld.

Figures 1 and 2 demonstrate the response of a .250" Driver/Pickup spot probe to flaws C1, C3, $\mathrm{C} 4$ and P5. A test frequency of $200 \mathrm{kHz}$ was used to achieve maximum sensitivity to the flaws and maintain adequate separation between flaw and noise responses. Driver pickup probes specifically designed for weld examinations (DTXP Cross Point Weld Scan Probes) were also used with similar results. This type of coil arrangement lends itself well to inspections involving heat affected zones and geometric changes associated with weld regions.

\section{"Free Span" Pitting:}

Figures 3 and 4 demonstrate the response of a .375" Driver/Pickup Spot Probe to the simulated near surface pitting. The pitting in this case is a series of round bottom holes machined into the top surface of the standard. These flaws are referred to as Flaw A through D. The graphics provided show the response of the coil operating at $50 \mathrm{kHz}$. As expected, we can demonstrate very good sensitivity to near surface pitting of this type.

It should also be noted that these pits can be detected from the far side of the plate standard. The assumption could be made that local areas of degradation would be detectable through the width of the part, ensuring $100 \%$ coverage.

\section{Corrosion / Wall Thinning:}

Using the 6 steps that Zetec machined into the standard, Figure 5 demonstrates the response of a .375 " Driver/Pickup spot probe to far surface corrosion type flaws. This test was performed at $7.5 \mathrm{kHz}$. The upper graphic was produced by nulling the probe on nominal material and placing it on each of the successive steps to show a change in the operating point. The bottom figure is a continuous scan over the same steps demonstrating a large area of corrosion or wall thinning.

\section{Future Development:}

All data presented has been manually collected and analyzed in a laboratory environment. It is foreseeable that the data acquisition and analysis could be streamlined using sophisticated software and hardware techniques. Using some of Zetec's existing technologies (i.e. software, testers and motor controllers) and focusing towards the goals set forth by INEL, I believe a system could be optimized to perform accurate and consistent scans of localized areas within the tanks. 
Further studies of flaw responses and coil configurations should be pursued to optimize results. At that time we could investigate further data analysis techniques such as phase and amplitude analysis for depth estimations.

\section{Summary / Conclusions:}

To date Zetec has performed a number of test to prove feasibility for this inspection. Everything we have done to date as part of the "no cost feasibility study" has proven conclusively the validity of the exam.

Based on the positive results, Zetec would be happy to pursue any aspect of the technique working in conjunction with INEL.

If you have any questions or comments on the testing or results, please feel free to contact me in the Probe Shop/Applications Department.

Best regards,

ZETEC, INC.

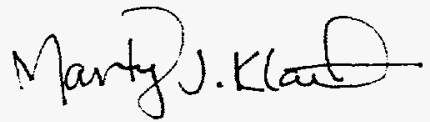

Marty J. Klatt

Applications Specialist

Attachments 

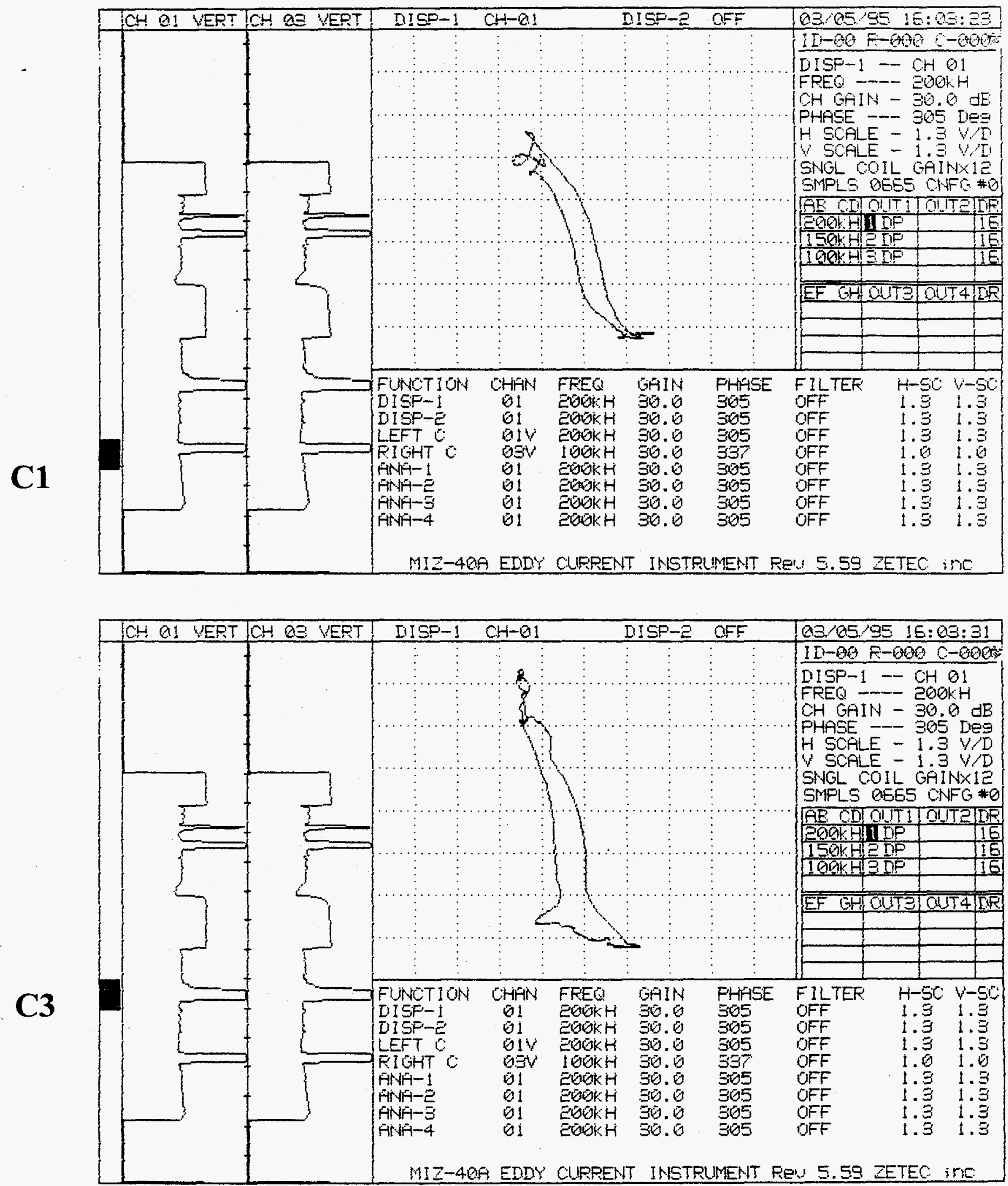

FIGURE 1 

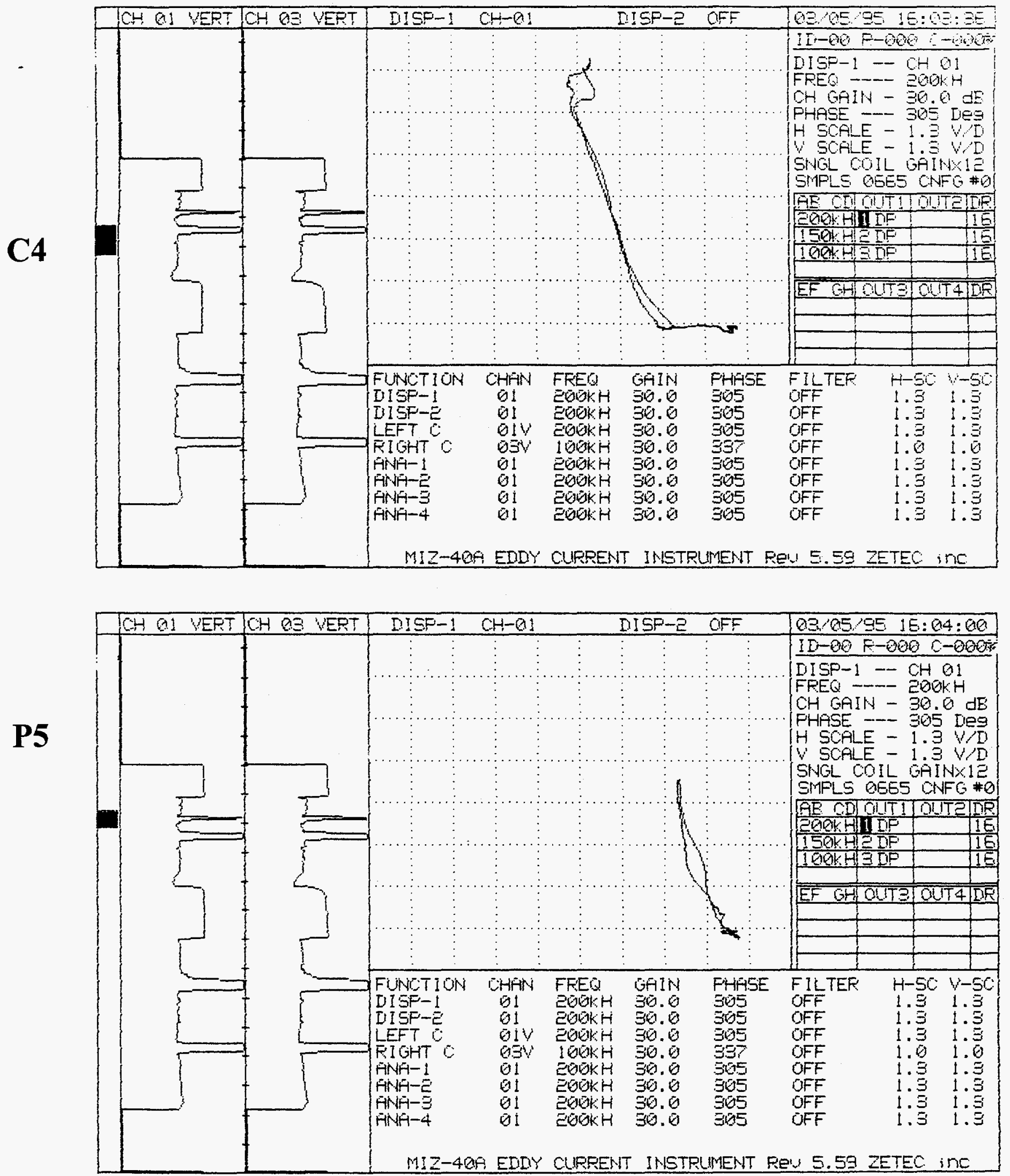

\section{FIGURE 2}



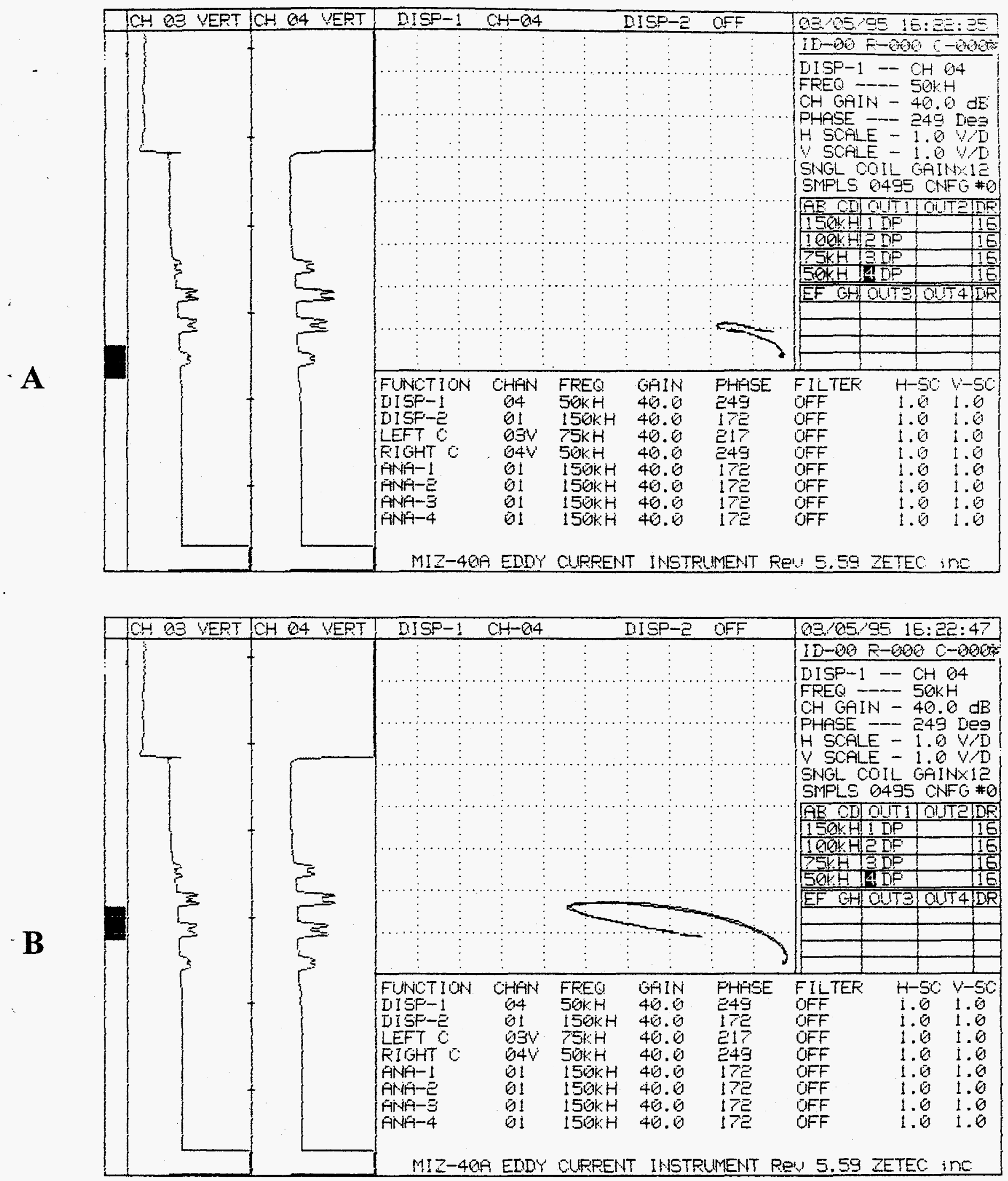

FIGURE 3 

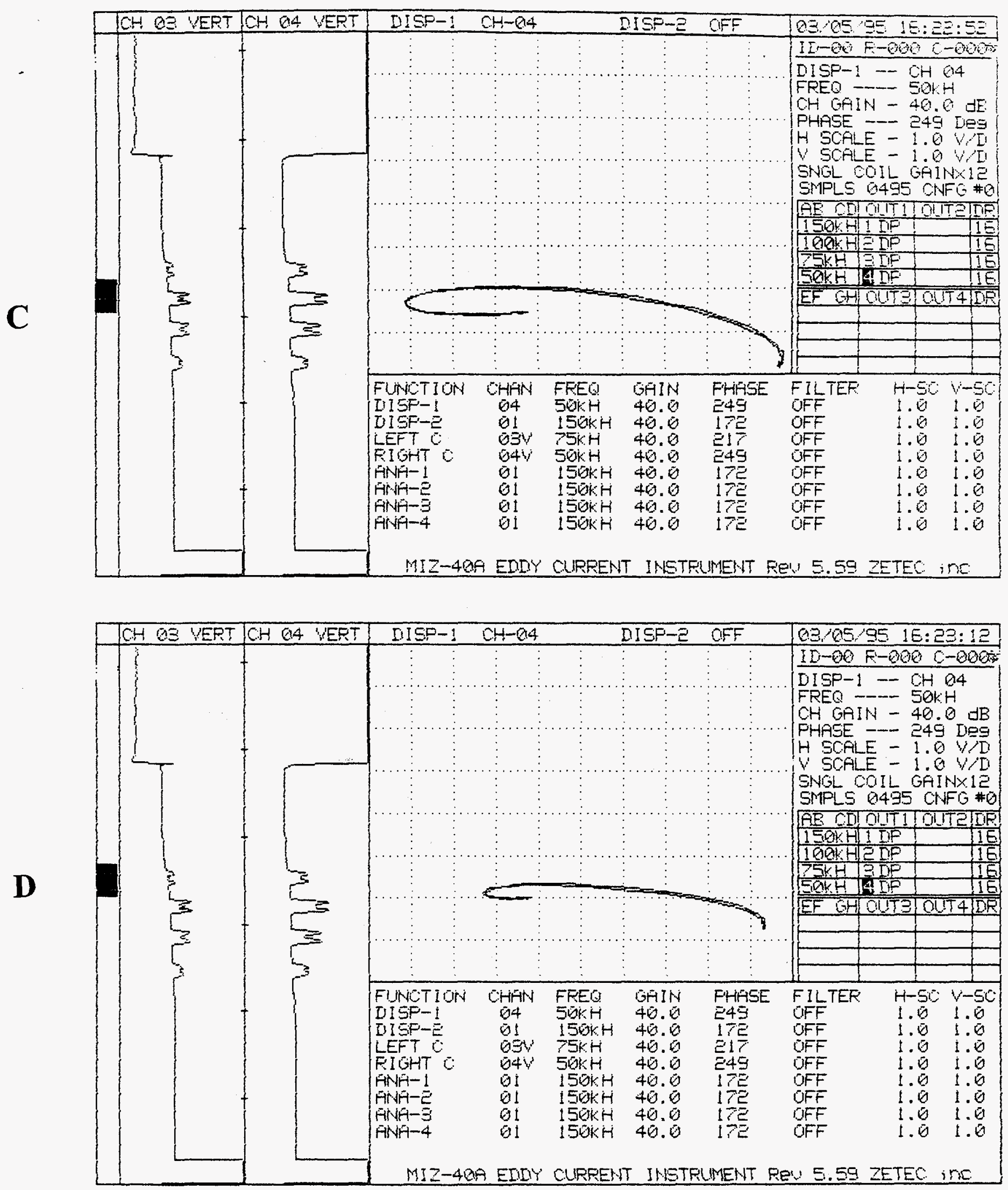

FIGURE 4 


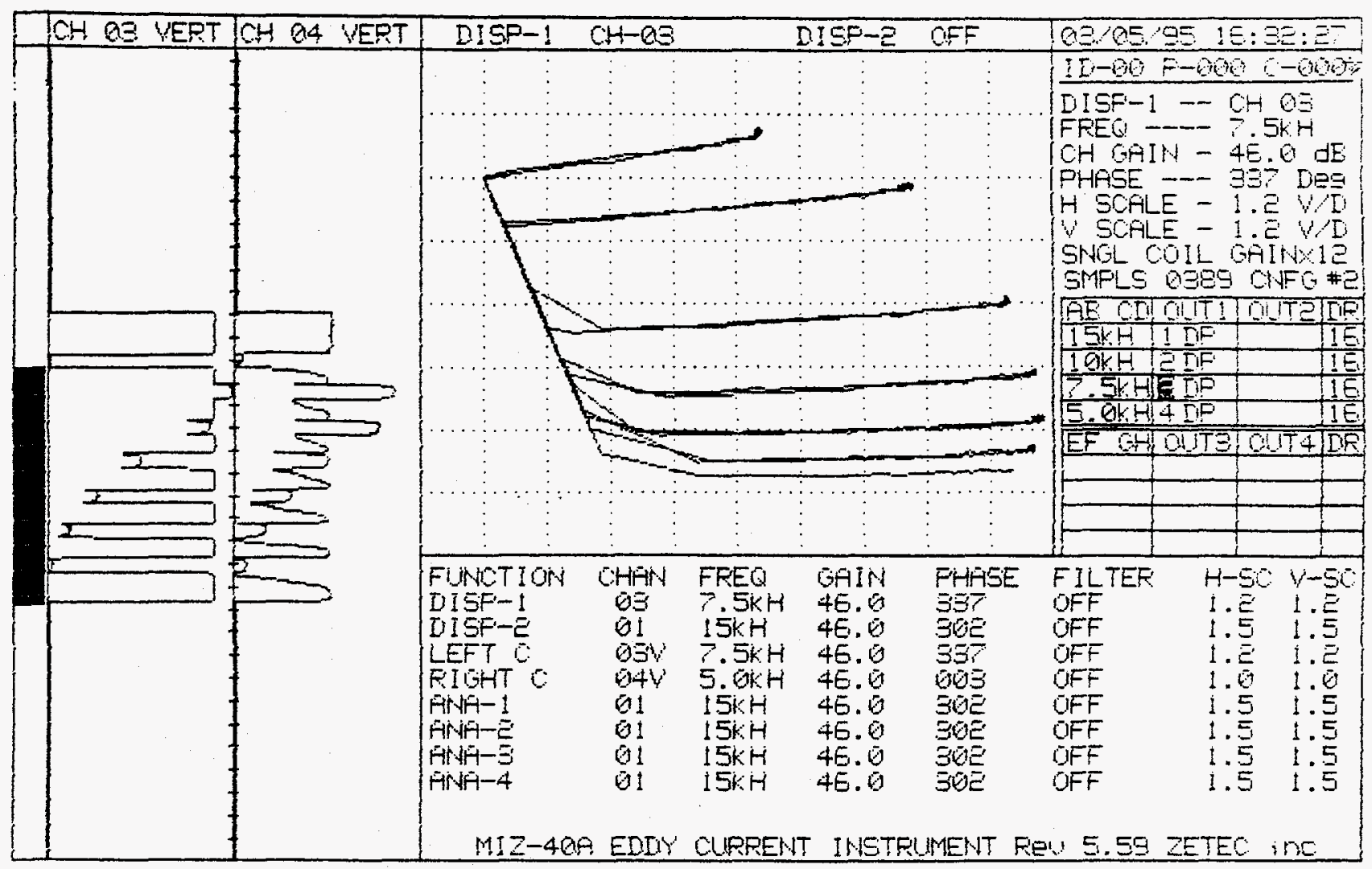

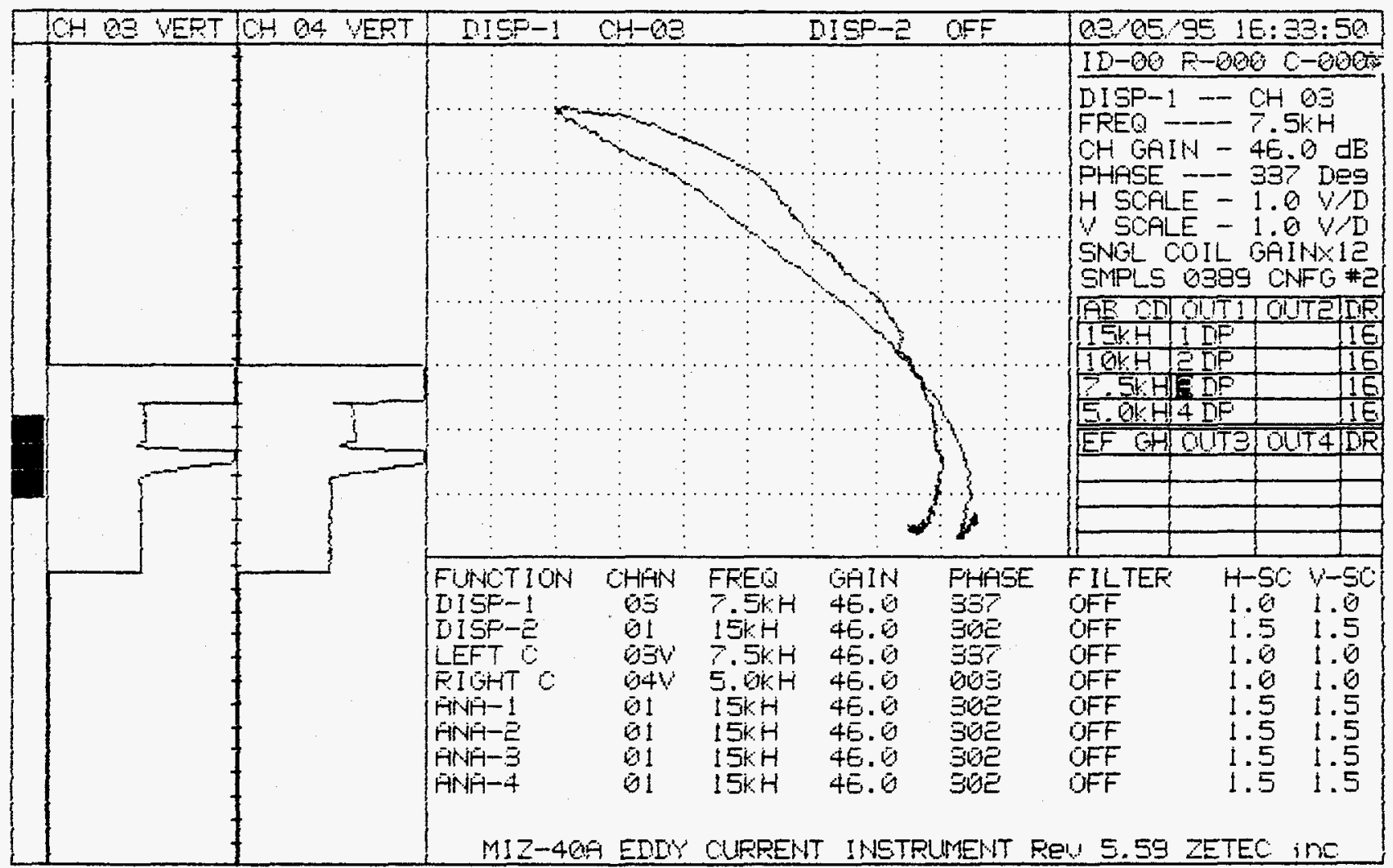

TOP - Steps E-J

Bottom - All Steps 


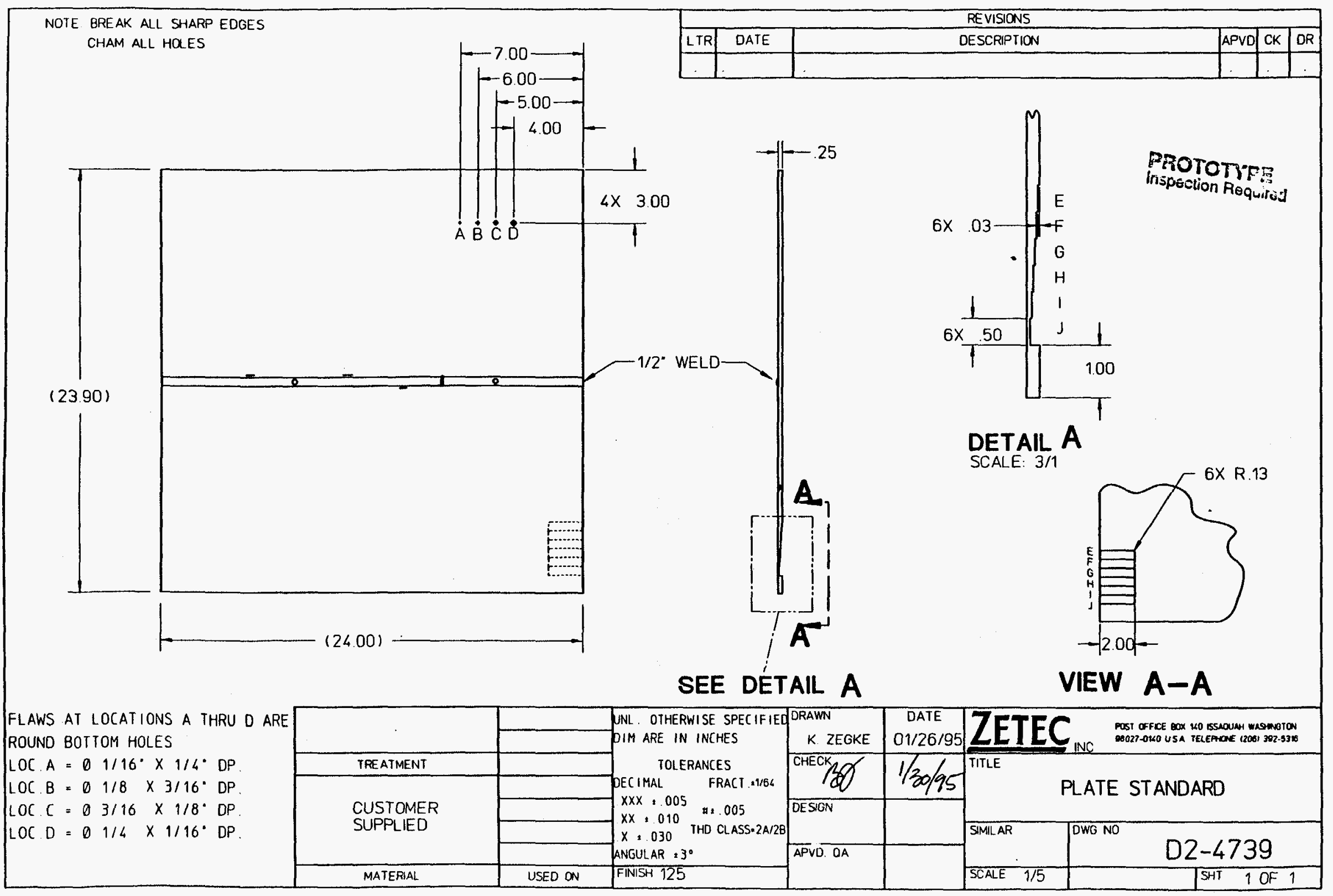

FIGURE 6 